\title{
La Red de Universidades Indígenas Interculturales y Comunitarias de Ab'ya Yala: La descolonización del pensamiento, la razón y el ser en Ab'ya Yala'
}

\author{
$A b^{\prime} y a$ Yala 's Network of Indigenous, Intercultural, and Community Universities: \\ The Thought Decolonization, Reason and Being in Ab'ya Yalala
}

\author{
Yuri Hamed Zapata Webb²
}

\section{Resumen}

En este artículo se han reflejado las razones del por qué se crea la Red de Universidades Indígenas Interculturales y Comunitarias de Ab'ya Yala (RUIICAY); se hizo primeramente un recorrido de sus miembros y cuáles son sus características filosóficas y epistemológicas, para luego centrarse en el camino que ha transitado desde su fundación en la búsqueda de establecer un modelo de Educación Superior, donde la Interculturalización es un eje primordial y esto comprende hacer instituciones educativas más inclusivas de las pluridiversidades.

Se han señalado las distintas actividades realizadas y por último se hace un apuntamiento hacia donde están dirigidos los esfuerzos de la RUIICAY en términos de descolonizar la educación para construir una sociedad más inclusiva.

Palabras clave: RUIICAY, Interculturalización de la Educación Superior, Descolonización del Pensamiento, La Razón, Epistemología, Procesos.

\section{Abstract}

In this article, it has been reflected the reasons why the Ab'ya Yala's Network of Indigenous, Intercultural and Community Universities (RUIICAY) was created; It was first a path of its members and learning what are their philosophical and epistemological characteristics, to then focus on the route that has transited since its foundation in the search to establish a model of Higher Education, where interculturalization is a fundamental axis and this includes making educational institutions more inclusive of pluridiversities.

The different activities carried out have been pointed out and, lastly, the RUIICAY's efforts are directed towards decolonizing education to build a more inclusive societyummary

Keywords: RUIICAY, Interculturalization of Higher Education, Decolonization of Thought, The Reason, Epistemology, Processes.

\footnotetext{
1 XIV Congreso Latinoamericano y del Caribe de Extensión Universitaria y Segundo Congreso Centroamericano de Compromiso Social para la vinculación social de la universidad con la sociedad, Managua 2017.

2 Candidato a Doctor en Educación Superior. Vicerrector General de la Universidad de las Regiones Autónomas de la Costa Caribe Nicaragüense. Correo: yuri. zapata@uraccan.edu.ni, ORCID: https://orcid.org/0000-0002-0209-4843
} 


\section{Introducción}

En este artículo esboza lo que es y hace la Red de Universidades Indígenas Interculturales Comunitarias de Ab'ya Yala (RUIICAY) en términos de desarrollar una propuesta de Educación Superior que trasciende los modelos occidentales que hoy existen en el mundo. La RUIICAY surge como una respuesta a los modelos de conquista, dominio, colonización y neocolonización que han vivido los pueblos afrodescendientes e Indígenas en nuestra Ab'ya Yala desde hace más de 500 años.

La conquista y el dominio para nuestras culturas significó avasallar nuestros espacios, robar nuestras riquezas, exterminar a las poblaciones, imposición de sus formas de vida de acuerdo y semejanza de la Europa Medieval, y los Estados Nacionales. Este es el impacto y el verdadero rostro del colonialismo salvaje que atentó en contra de la dignidad, la vida y la existencia misma.

\section{En este impacto sobresalen:}

1. El desconocimiento de nuestros nombres y división sociogeográfica, imponiendo otras denominaciones a nuestros espacios geográficos y concentraciones poblacionales. Además de bautizarnos con nombres que nos hicieron perder nuestra identidad de pueblos.

2. La pérdida de culturas y lenguas por la imposición de las propias de Europa. Esto implicó incluso, la desaparición de culturas milenarias al ser destruidas las bases humanas y materiales que las sustentaban.

3. Se introdujeron nuevas enfermedades que diezmaron a la población por no tener inmunidad a las mismas.

4. El desconocimiento hacia las formas propias de construir conocimientos y su difusión entre los pueblos. Se dio la destrucción de los conocimientos y se impuso la "ciencia de occidente", sobre los conocimientos, saberes y prácticas ancestrales de los pueblos.

Por otro lado, al darse la colonización y la neocolonización, se sucedieron procesos de imposición en el orden:

1. Político: Implicó el establecimiento de los Estados Nacionales, regidos por leyes y normas a la usanza de las potencias coloniales y neocoloniales.

2. Socio-Organizacional: Que establecía nuevas formas de estructuración de la sociedad: división de clases sociales, sistemas organizativos de los núcleos habitacionales y de la población.

3. Epistémica: Al darse la negación y descalificando los sistemas de conocimientos de las civilizaciones y los pueblos de Ab'ya Yala, que han aportado grandemente al conocimiento universal, superponiendo las "verdades absolutas de las ciencias" occidentales.

4. Mono Cultural: Refiere la imposición de un tipo de cultura, la blanca europea sobre las diversidades de pueblos existentes, cada uno con sus propias formas de vida.

5. Cosmogónica: Dada por la imposición de una forma de ver al mundo, desde el cristianismo y las ciencias, negando las formas diversas de los pueblos y su relación con la naturaleza y el cosmos.

6. Económica: Determinación de un sistema económico y productivo extractivo y para crear plusvalía sólo para un sector poblacional.

7. Educativa: Se impuso la educación desde el punto de vista de los Estados coloniales y neocoloniales, negando e invisibilizando la diversidad de pueblos e historias. 
A su vez, la colonización y neocolonización a través de modelos económicos como la globalización, han impuesto una división geográfica: i) Norte-Sur, ii) Este-Oeste. División que tiene como objetivos desvirtuar los aportes de los pueblos y crear una perspectiva geográfica de desarrollo y de conocimientos donde se da la lucha por hacer prevalecer el "conocimiento científico" de las sociedades del Norte y el Este, contra el conocimiento y la práctica "empírica de los pueblos" del Sur y del Oeste. En otras palabras, se mantiene la visión neocolonizante de la imposición de la geopolítica del conocimiento y de pueblos y culturas "desarrolladas y no desarrolladas".

Lo anterior determinó que se establecieran modelos educativos que no respondían a las particularidades de los pueblos, por lo que después de siglos de resistencia de los pueblos afrodescendientes e indígenas, se trata de establecer instituciones educativas que atiendan las particularidades de la historia, la identidad, la espiritualidad de los pueblos y sus procesos de desarrollo; elementos que son expuestos en este artículo que es escrito la base de los procesos de socialización de sus miembros en los distintos espacios de trabajo.

\section{Desarrollo}

\section{¿Quiénes Somos, ¿qué nos caracteriza y cuál es la finalidad?}

Somos la Red de Universidades Indígenas Interculturales y Comuntarias de Ab'ya Yala que nos hemos creado desde las raíces culturales y cosmogónicas de los pueblos, para constituirnos en servidores educativos por el buen vivir y la construcción de ciudadanías interculturales de los pueblos en estrecha armonía con la naturaleza y la gran madre tierra (la Pachamama).

La RUIICAY está conformada por 11 instituciones de diversos países. Entre estos están:

\begin{tabular}{|l|l|}
\hline \multicolumn{1}{|c|}{ Institución Educativa } & País \\
\hline Universidad de las Regiones Autónomas de la Costa Caribe Nicaragüense. URACCAN & NICARAGUA \\
\hline Pluriversidad Amawtay Wasi & Ecuador \\
\hline Universidad Autónoma Indígena Intercultural UAIIN & Colombia \\
\hline Universidad Boliviana Aymara & Bolivia \\
\hline Universidad Boliviana Quechua & Bolivia \\
\hline Universidad Boliviana Guaraní & Bolivia \\
\hline Universidad de los Pueblos del Sur, UNISUR & México \\
\hline Oficina Pueblos Indígenas-Universidad de Panamá (OPINUP) & Panamá \\
\hline Instituto de Educación Superior Intercultural, Campinta Guazú, Gloria Peréz & Argentina \\
\hline Universidad IXIL & Guatemala \\
\hline Universidad de Venezuela & Venezuela \\
\hline
\end{tabular}


Cada institución educativa cuenta con su respaldo jurídico nacional e internacional que determina la posibilidad de establecer el modelo propio de universidad y las metodologías que recojan los sistemas de conocimientos, saberes y prácticas de los pueblos.

\section{Nuestras instituciones se caracterizan por:}

1. Ser parte de las naciones originarias e indígenas, pueblos afrodescendientes y comunidades étnicas.

2. Estar articuladas y vinculados a los movimientos indígenas y sociales de cada país.

3. Ser el espacio de recuperación, fortalecimiento y acompañamiento de los planes y propuestas de vida de los pueblos y sus organizaciones para impulsar el buen vivir, el vivir bien y el vivir con dignidad.

4. Establecer la vinculación teórica práctica en el desarrollo de los procesos de aprendizajes, creación y recreación comunitaria de saberes, fundamentados desde el conocimiento ancestral.

5. Implementar metodologías que facilitan el diálogo e interacción de los diferentes saberes.

6. Buscar la armonía entre la razón y el corazón para la solución de los problemas de formación, en tanto se fundamente en el sentir, pensar y actuar en un contexto de diversidad.

7. Hacer uso de la lengua materna en los procesos y programas de aprendizajes, creación y recreación comunitaria de saberes.

8. Meditamos, reflexionamos, trascendemos y hacemos desde lo propio para visibilizar lo nuestro.

\section{Nuestra finalidad está orientada a:}

1. Crear un Sistema de Educación Superior Intercultural Continental con identidad, dentro de un marco de integralidad del conocimiento que permita superar la ruptura entre la práctica y la teoría del conocimiento.

2. Impulsar y fortalecer las instituciones de Educación Superior Intercultural, indígena y comunitaria en el marco de los Estados Nacionales.

3. Determinar un posicionamiento político y legislativo en torno al desarrollo con identidad de los pueblos y la construcción de una nueva sociedad y ciudadanía intercultural convivencial.

4. Promover un sistema de evaluación y acreditación de los centros del conocimiento mayor indígena, intercultural y comunitario y de sus modelos educativos.

5. Impulsar sistemáticamente procesos investigativos y de emprendimiento con los componentes dialógicos, conceptuales con rigurosidad y profundidad.

6. Sistematización y publicación de los procesos de recreación y generación de conocimientos que aporten al buen vivir de los pueblos.

\section{Nuestra Minga (Visión) y responsabilidad social (Misión)}

\section{Al constituirnos como red, consensuamos qué nos guía y es lo siguiente:}

\section{Minga}

Las Universidades Interculturales de Ab'ya Yala, son el cimiento de las comunidades científicas interculturales que a través de programas y procesos educativos y de incidencia sociopolítica contribuyen a la construcción y promoción de ciudadanías interculturales y el buen vivir de los pueblos (RUIICAY, 2018).

\section{Responsabilidad social}

Las Universidades Interculturales de Ab'ya Yala, centramos nuestros esfuerzos en la formación de talentos humanos, con conocimientos y capacidades científico-técnica, actitudes interculturales, humanistas, sentido del emprendimiento y la innovación, para establecer la relación armónica entre la naturaleza y la sociedad para el desarrollo con identidad y el buen vivir de los Pueblos de Ab'ya Yala. (RUIICAY, 2018). 
Además, definimos que nuestra responsabilidad social, como instituciones académicas que trabajan en función de:

1. Formación de talentos humanos, a través de procesos educativos pertinentes, inclusivos y de calidad.

2. Promoción de actitudes interculturales, que permitan la convivencia armónica de los pueblos y sus culturas.

3. Relación armónica entre la naturaleza y la sociedad, que permita la sustentabilidad y sostenibilidad de la humanidad y el medio ambiente.

4. Desarrollo con identidad y el buen vivir de los Pueblos de Ab'ya Yala a.

Estos elementos, se complementan con los ejes transversales con los cuales se avanza para construir sociedades interculturales.

1. La identidad étnica y cultural que permite el empoderamiento de los pueblos.

2. La interculturalidad y cosmovisiones que determinan los distintos sistemas de conocimientos y espiritualidades que le dan explicación a los fenómenos y procesos sociales y naturales.

3. La igualdad de derechos y oportunidades que procura hacer justicia ante las desigualdades de género, de pueblos, culturas y áreas geográficas.

4. La unidad continental que propicia el hermanamiento de los pueblos por una causa común, el desarrollo con identidad para el buen vivir de los pueblos y la construcción de ciudadanías interculturales.

5. El medio ambiente y desarrollo sostenible como alternativa de los pueblos para la pervivencia en el tiempo.

6. La formación de recursos humanos de manera sistemática y para toda la vida, lo que permite el mejoramiento del buen vivir de los pueblos.

7. La construcción y recreación de conocimientos mediante el diálogo de saberes desde cada proceso que desarrolla la universidad.

\section{Filosofía y episteme de la RUIICAY}

De acuerdo a nuestros marcos jurídicos nacionales y considerando nuestras propias normas, políticas y estatutos como Instituciones Indígenas, Interculturales de Educación Superior (IIICES), determinamos, guiarnos por los preceptos a saber: (RUIICAY, 2018)

1. Descolonización, intraculturalidad, interculturalidad que permite reconstruir la historia de los pueblos y su relación con los Estados y Gobiernos en función de establecer sociedades armónicas.

2. Educación productiva, comunitaria y familiar, orientada a rescatar los valores de convivencia y desarrollo de procesos a través de prácticas productivas que permitan el bienestar de la población.

3. Comunitarismo organizacional: Democracia comunitaria, lo que permite la unidad en la diversidad en la construcción de los procesos de desarrollo en lo político, social, cultural, económico y ambiental, dónde cada mujer y hombre tienen la posibilidad de participar en los procesos de toma de decisiones de la comunidad.

4. Modelo productivo comunitario, orientado a asegurar la soberanía y seguridad alimentaria de la población, sobre la base del rescate, promoción de los alimentos y semillas tradicionales, así como el uso de plantas medicinales para enfrentar los procesos de salud y enfermedad.

5. Restitución tejido social vivo: Articulación, integración universidad, sociedad y Gobierno-Estado, desde una óptica de relaciones de igualdad y de respeto a las formas propias de los pueblos para el impulso de procesos de desarrollo con identidad para el buen vivir. 


\section{Acciones que desarrolla la RUIICAY}

En nuestro caminar como red, hemos impulsado procesos que están encaminados a (RUIICAY, 2017):

1. La construcción y desarrollo de Programas de Maestrías Comunes: Comunicación Intercultural, Educación Intercultural Multilingüe, Paradigmas de Buen Vivir con énfasis en cambio climático.

2. La acreditación de títulos de licenciaturas que se sirven en las universidades miembros de la red.

3. La facilitación de la movilidad académica de docentes y estudiantes entre las universidades miembros de la red.

4. El Reconocimiento a los y las portadoras de conocimientos, saberes, haceres y su inserción en los procesos de las comunidades de aprendizajes en los distintos niveles de formación.

5. Compartimos las filosofías y cosmovisiones de los pueblos en las aulas de clase como una manera de revitalizar las culturas, lenguas e historias de los pueblos y su aporte a las sociedades multiculturales.

6. Hacemos reflexiones interculturales sobre la Educación Superior a nivel continental para posicionar la idea de modelos educativos que responsan a las diversas matrices culturales y se hagan efectivas las premisas del desarrollo con identidad y pertinente a los contextos.

7. Construimos nuestro Sistema de Acreditación y Reconocimiento de las IIICES para que nuestras instituciones se valoren de acuerdo con sus particularidades filosóficas y epistémicas.

8. Construimos y acompañamos los planes de vida de los pueblos para que estos puedan ser los sujetos activos en sus procesos de transformación para la construcción de las ciudadanías interculturales.

La orientación de nuestros procesos

La RUIICAY tiene orientado su camino a asegurar los procesos siguientes: (RUIICAY, 2018)

\section{a. Descolonizar en lo educativo}

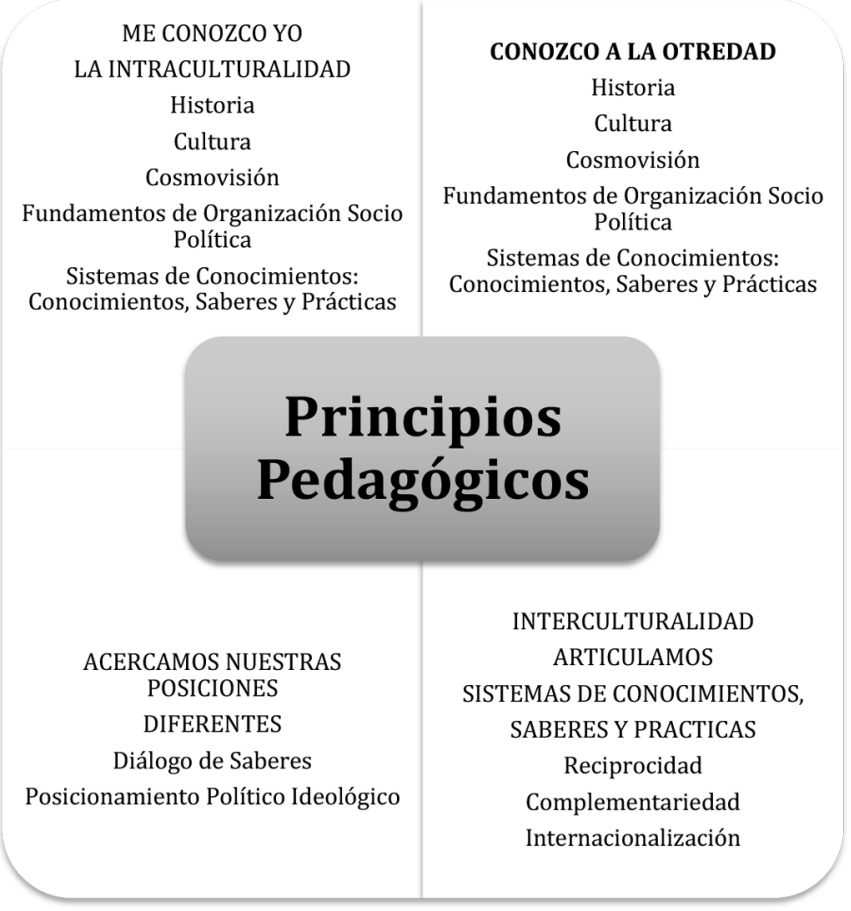

Figura 1: Descolonización educativo. 


\section{b. Generación de conocimientos}

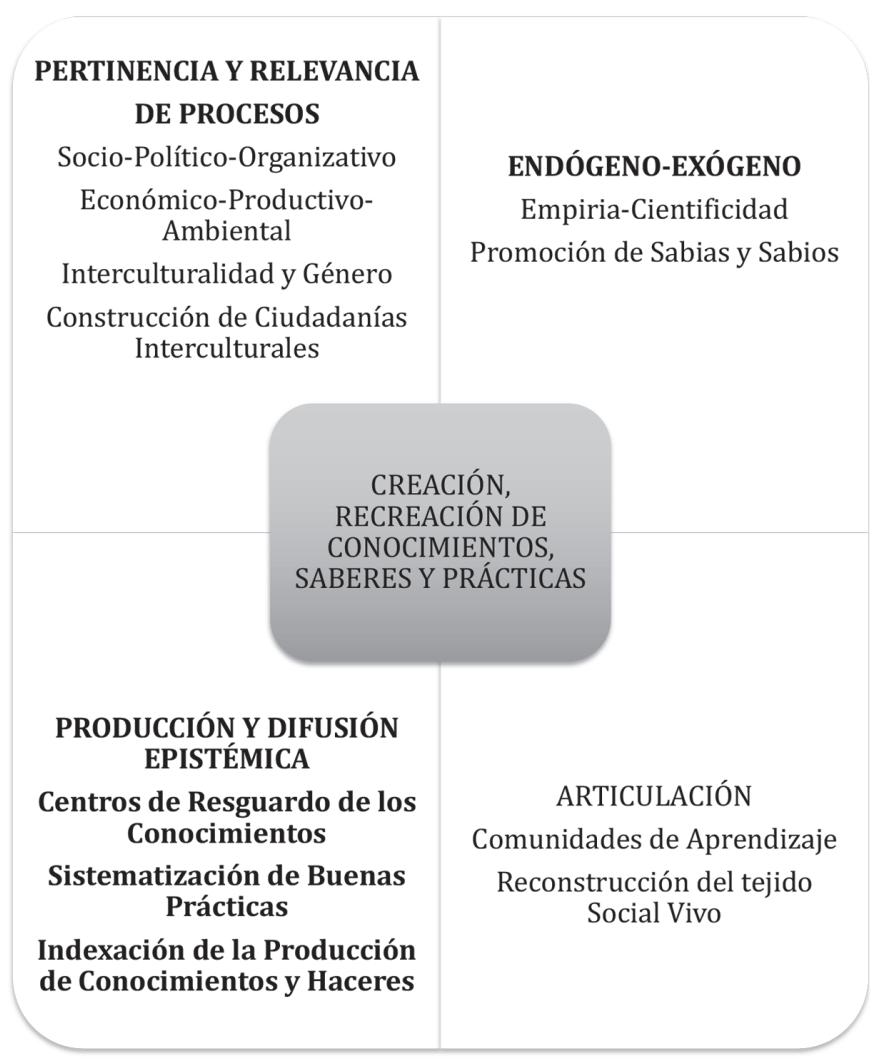

Figura 2: Generación de conocimientos

\section{c. Articulación del tejido social}

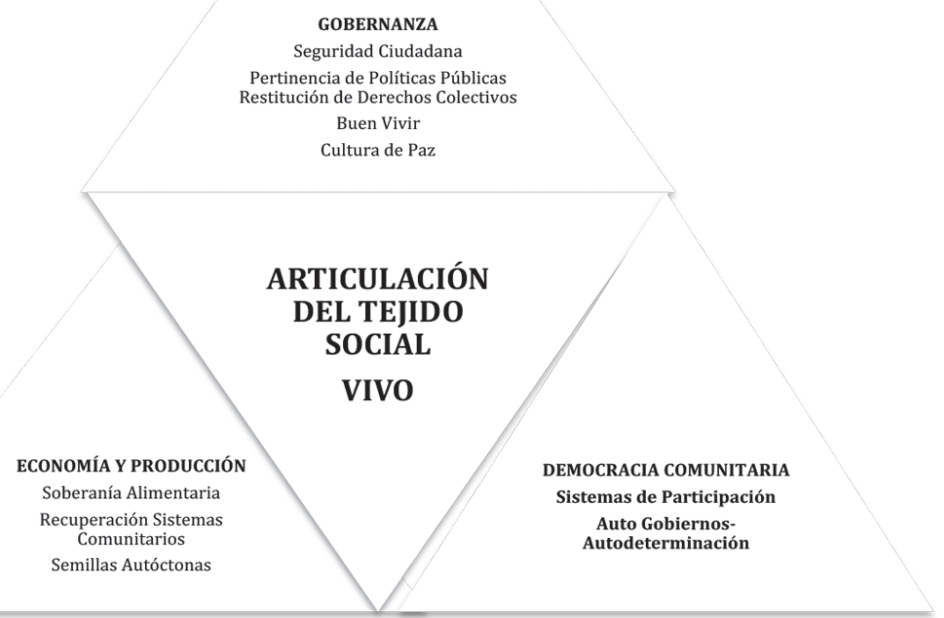

Figura 3: Articulación del tejido social 
Con estos procesos en desarrollo desde nuestras IIICES, en Ab'ya Yala se propugna por un modelo educativo que lleve a:

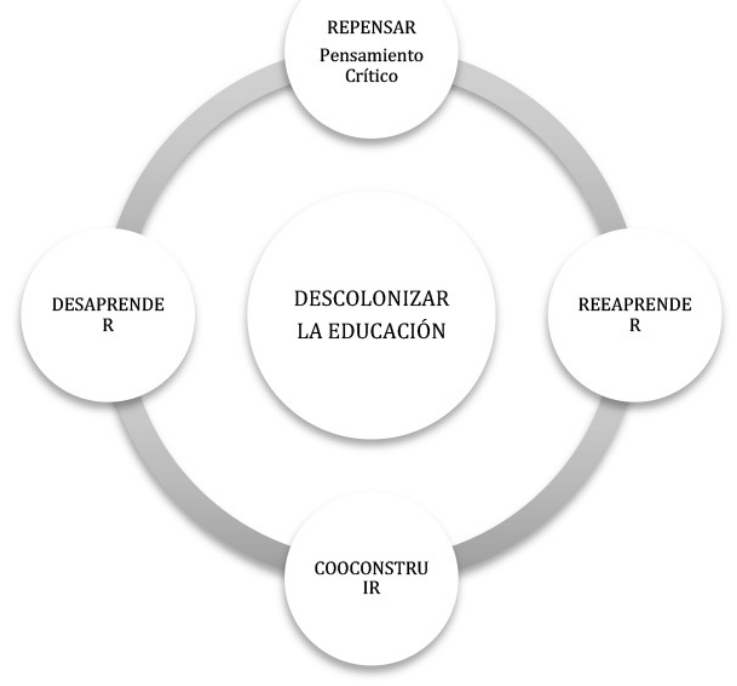

Figura: 4: modelo educativo descolonizar de la educación

\section{Pero para hablar de la descolonización se debe estar claro que este proceso implica:}

1. Creer en la pluralidad de sistemas epistemológicos: Hacer un reconocimiento a la emergencia de nuevas epistemologías: Epistemologías del Sur.

2. Interculturalizar la educación y formación: Articular y promover los distintos sistemas de conocimientos para potenciar las identidades y culturas de los pueblos y con ello su aporte a la universalidad.

3. Potenciar las voces de los no oídos e invisibilizados para se conviertan en los voceros de su propio desarrollo.

4. Ejercicio efectivo de la autodeterminación: Las autonomías de los pueblos para construir su camino para el buen vivir.

5. Co-construir las propias historias de los pueblos y visibilizar los aportes a la humanidad.

6. Aprender a indagar contextualmente para la transformación real de la sociedad y hacer propuestas pertinentes desde los pueblos y sus culturas.

\section{Conclusiones}

\section{Entre las principales conclusiones encontramos que:}

1. Las Universidades miembro de la RUIICAY, son instituciones académicas que tienen como fundamento la cosmovisión de los pueblos y sobre esa base construyen su modelo institucional educativo.

2. La RUIICAY ha venido construyendo un camino para que la Educación Superior se interculturalice en los países de incidencia.

3. El paradigma epistemológico y filosófico de la RUIICAY está orientado a la descolonización de los procesos educativos, la construcción del buen vivir de los pueblos y el establecimiento de ciudadanías interculturales. 


\section{Lista de referencias}

Red de Universidades Indígenas Interculturales y Comunitarias de Abya Yala (RUIICA, 2010). Acta Constitutiva de la Red. La Paz Bolivia, 21 y 22 junio.

Red de Universidades Indígenas Interculturales y Comunitarias de Abya Yala (RUIICAy ,2010). Fundamentos de la Red. La Paz Bolivia, 21 y 22 de junio.

RUIICAY (2017). El Caminar de la RUIICAY en la Construcción de la Educación Superior Intercultural. Octubre, Quito, Ecuador, Abya Yala.

RUIICAY (2017). Hacia la CRES 2018. Posicionamiento de la Red de Universidades Indígenas Interculturales Comunitarias de Abya Yala. San José de Puembo, Ecuador, 23 noviembre.

RUIICAY (2018). La Decolonización del Pensamiento y la Razón en Abya Yala. Documento de Trabajo. 